\title{
Uterine Artery Embolization (UAE) for Fibroid Treatment - Results of the 5th Radiological Gynecological Expert Meeting
}

\author{
Uterusarterienembolisation (UAE) zur Myombehandlung - \\ Ergebnisse des 5. radiologisch-gynäkologischen Expertentreffens
}

Authors

Affiliations
T. Kröncke ${ }^{1}$, M. David ${ }^{2}$

Department of Diagnostic and Interventional Radiology and Neuroradiology, Klinikum Augsburg, Germany

2 Department of Gynecology, Charité Universitätsmedizin Berlin, Germany
Key words

0 uterus

embolization

- leiomyoma

received $\quad 1.3 .2015$

accepted $\quad 6.3 .2015$

Bibliography

DOI http://dx.doi.org/ 10.1055/s-0034-1399345

Published online: 22.4.2015

Fortschr Röntgenstr 2015; 187:

483-485 ๑ Georg Thieme

Verlag KG Stuttgart · New York . ISSN 1438-9029

\section{Correspondence}

PD Dr. med. Thomas Kröncke, MBA

Department of Diagnostic and Interventional Radiology and Neuroradiology, Klinikum

Augsburg

Stenglinstr. 2

86156 Augsburg

Germany

Tel.: ++49/821/4002441

Fax: ++ 49/821/4003312

thomas.kroencke@klinikum-

augsburg.de

Prof. Dr. med. Matthias David

Department of Gynecology,

Charité Universitätsmedizin

Berlin, Campus Virchow-

Klinikum

Augustenburger Platz 1

13353 Berlin

Germany

\section{Preamble}

$\nabla$

Uterine artery embolization (UAE) is an established, organ-preserving, safe and effective procedure in the therapeutic spectrum treatment of fibroid-related symptoms.

The aim of UAE is the reduction or elimination of fibroid-related symptoms, but not the removal of the myoma. However, it also accomplishes a reduction of the size of the fibroid.

There is a consensus between the disciplines of gynecology and radiology that an indication for treatment of uterine fibroids (leiomyoma) should be made after examination and consultation of a gynecologist. Comprehensive advice regarding treatment options for symptomatic uterine fibroids encompasses not only medical treatment and surgical options, but also non-surgical therapy options explicitly including uterine artery embolization. The decision for or against an alternative therapy should be made taking into account the patient's desire for, and knowledge of, therapeutic alternatives, their chances of success and limitations, as well as typical side effects and possible complications (informed decision).

Uterine artery embolization is a therapeutic procedure which is available in Germany, Austria and Switzerland for patients with fibroid-related symptoms and provides further individualization of therapy in cases of uterine fibroids.

\footnotetext{
* On behalf of the participants of the Consensus Meeting.
}

\section{Aim of the consensus meeting \\ $\nabla$}

The intention of the consensus meeting was the current assessment of UAE. The participants in the meeting of radiological-gynecological experts, after renewed evaluation of existing literature, internationally published recommendations and their own experience, and after extensive discussion, came to a consensus between the two disciplines. The panel of experts was aware that this was an assessment of the possibilities and limits of radiological therapy held in conjunction with specialists in gynecology who do not perform the procedure themselves, but who possess relevant experience in the diagnosis and treatment of disorders of female genital organs. The group of experts composed of 13 radiologists and 12 gynecologists met on January 17 , 2015 in Berlin for the fifth radiological-gynecological consensus meeting included gynecologists from Switzerland and Austria. After extensive - and somewhat contentious - discussion, the group came to a consensus regarding the following recommendations. The consensus statement is supported by the gynecologists and radiologists listed at the end of this work. The paper reflects the current state of knowledge.

\section{Structural prerequisites and quality assurance for the performance of UAE $\nabla$}

Uterine artery embolization should be performed only at those clinics where gynecologists and radiologists possess the required expertise in performing UAE, where adequate and structured pain therapy and management of side effects are in place and which have competence in conservative and surgical therapy of fibroids. 
In particular, due to required post-interventional pain therapy, UAE should be performed on an in-patient basis in clinics.

Prior to introducing UAE to a clinic, it is recommended that theoretical and practical instruction be obtained at a center with extensive experience in performing the procedure. In addition to legally-required documentation, quality assurance should also include critical review and optimization of the radiation exposure parameters (area dose product, fluoroscopy time) for UAE.

Participation in a suitable quality assurance supported by the professional associations is recommended.

\section{Examinations required prior to UAE \\ $\nabla$}

The choice of therapy should be based on an examination performed by a gynecologist including vaginal and/or abdominal ultrasound (depending on the size of the uterine fibroids). An MRI examination is strongly recommended if ultrasound does not offer a definite imaging diagnosis.

Prior to each fibroid embolization the indications for hysteroscopy D \& C must be reviewed. Unremarkable cytological smear findings of the uterine cervix must have been obtained within the previous year.

In addition to a pregnancy test, the following laboratory values must be obtained: creatinine, coagulation profile, thyroid values (if there is positive history for thyroid disease), a complete blood count and CRP. An active genitourinary inflammation must be excluded, based on past medical history and symptomology.

Likewise, prior to UAE, within the context of the obtaining informed consent, the patient should be informed regarding the absence of pre-interventional histological confirmation of presumed uterine fibroids, as is the case with all other organ-conserving fibroid therapies (see also the statement of the DGGG [German Society of Gynecology and Obstetrics] "Implementation of the Medical Devices Act - Gynecological Morcellators" dated 8/29/2014).

\section{Indications for UAE}

$\nabla$

A symptomatic uterine fibroid is an indication for uterine artery embolization. UAE represents an alternative to surgical and medication-based procedures as well fibroid treatment using focused ultrasound, regardless of the size and numbers of the fibroids or previous surgery. The basis for the choice of therapy should be the aim of the treatment as well as the patient's desire.

\section{Success criteria for UAE}

$\nabla$

The focus of the assessment of the therapeutic success after UAE is primarily on the improvement or complete disappearance of (fibroid-related) symptoms indicated by the patient, and less on the volume reduction of a dominant fibroid or of the entire uterus after treatment.

\section{Contraindications for UAE}

$\nabla$

Technical

Relative

- Prior GnRH analogues treatment during the previous 3 months (increased risk of vasospasm of the uterine artery)

- According to current knowledge, administration of ulipristal acetate has no significance for the diagnosis and performance of the UAE, and will not affect the results of uterine artery embolization

\section{Anatomical}

Relative

- Isolated submucosal fibroids types 0 and I according to ESGE (European Society for Gynaecological Endoscopy), accessible for hysteroscopic ablation.

- Isolated pedunculated subserosal fibroids

- Fibroid supplied by an ovarian artery; it is necessary to weigh the risks and benefits of an additive embolization of the related ovarian artery

\section{Clinical considerations}

Absolute

- Suspicion of malignancy

- Pregnancy

- Acute genital infection

- Overt hyperthyroidism/florid thyroiditis with hyperthyroid metabolism as well as planned or ongoing radiotherapy if iodine-based contrast medium is used

Relative

- Documented allergic reaction to iodine-based contrast media

- Patient is in post-menopause

- Allergic reaction to local anesthesia

- Latent hyperthyroidism

- Renal insufficiency

- Document wish to conceive in the near future

\section{UAE for patients wishing to become pregnant}

In the context of fertility treatment, UAE is considered to be at best a last resort. Possible risks primarily include potential reduction of the ovarian reserve, increased risk of miscarriage, impaired placentation and postpartum hemorrhage.

The role of UAE as a treatment option for patients with symptomatic uterine fibroids wishing to become pregnant has not been sufficiently clarified in the existing literature. The possibility of UAE should be reviewed in the case of a patient with extensive uterine fibroid disease and and a desire to conceive and for whom hysterectomy is considered.

\section{Preoperative fibroid embolization as exception (PUAE) $\nabla$}

Preoperative uterine artery embolization as direct preparation for surgical myomectomy can be considered individually for such patients who absolutely wish to retain their uterus, but for whom there is a significantly high risk of hemorrhage and/or for whom there is nevertheless a risk of a 
required hysterectomy "for technical reasons" (such as fibroids that are very large, hard to remove or fibroids in a difficult location).

\section{Radiation protection}

Radiation protection is an important issue with respect to UAE. Pulsed fluoroscopy should be employed. Serial angiographic runs as well as oblique projections should be kept to a minimum. As a rule, an imaging frequency of 1 image per second suffices. Under normal conditions, average values for the dose area product should lie below $50 \mathrm{~Gy} \times \mathrm{cm}^{2}$ (corresponding to $5000 \mathrm{cGy} \times \mathrm{cm}^{2}$ or $5000 \mu \mathrm{Gy} \times \mathrm{m}^{2}$ ) for pulsed systems. Adhering to these recommendations radiation exposure is in the range of 2 to 3 CT examinations of the abdomen.

\section{Side effects \\ $\nabla$}

Related side effects and complications of UAE include: postembolization syndrome, amenorrhea as a result of impairment or failure of ovarian function, pain, discharge, angiography-related complications (e.g. inguinal hematoma), vaginal discharge of fibroid material, hot flashes, endometritis/myometritis, deep leg vein thrombosis/pulmonary embolus.

Uterine discharge within the first few weeks after UAE can be normal. In case of abnormal or purulent discharge, measures to exclude and treat possible infections should be initiated. Menorrhagia, cramping lower abdominal pain or discharge of tissue can primarily appear in cases of submucosal fibroids. Depending on the symptoms and the findings of diagnostic imaging, hysteroscopic fibroid resection or transvaginal removal of a fibroid may be indicated likewise to the treatment indicated in cases of spontaneous fibroid passage or expulsion. Hysterectomy is not indicated a priori. In cases of doubt, the center performing the UAE should be consulted.

\section{Post-treatment examination after UAE \\ $\nabla$}

Post-treatment examination by a specialist is recommended approx. 6 months after UAE. Imaging procedures are useful (i.e. sonography in conjunction with Doppler ultrasound, MRI). Further clarification is required if the therapy is unsuccessful (no improvement of symptoms and/or size progression of the fibroid), or imaging reveals unexpected findings (increase in size of myoma(s)) or uterus and/or no devascularization of the fibroid(s).

\section{Outlook}

\section{$\nabla$}

Based upon available data and experience, a revision of recommendations for uterine artery embolization of fibroidrelated symptoms is planned for 2017.

\section{Meeting participants \\ $\nabla$}

Prof. Dr.med. Babür Aydeniz/Ingolstadt

Dr. med. Michael Bartsch/Hamburg

Dr. med. Tobias Belting/Munich

Prof. Dr. med. Markus Düx/Frankfurt a. M.

Prof. Dr. med. Dr. phil. Andreas Ebert/Berlin

Dr. med. Karoline Heinrich/Munich

Dr. med. Thomas Hess/Winterthur (Switzerland)

Dr. med. Friederike Hoellen/Lübeck

PD Dr. med. Peter Landwehr/Hannover

Prof. Dr. med. Werner Neuhaus/Cologne

Dr. med. Zbigniew Owsianowski/Jena

Prof. Dr. med. Boris Radeleff/Heidelberg

Prof. Dr. med. Götz Richter/Stuttgart

PD Dr. med. Stefan Rimbach/Feldkirch (Austria)

Prof. Dr. med. Thomas Pfammatter/Zürich (Switzerland)

Dr. med. Gernot Rott/Duisburg

PD Dr. med. Christian Scheurig-Münkler/Berlin

Dr. med. Renana Schinker/Hamburg

Dr. med. Wolfram Seifert/Gehrden

PD Dr. med. Wulf Siggelkow/Hannover

Prof. Dr. med. Ulf Teichgräber/Jena

Dr. med. Götz Voshage/Gehrden

PD Dr. Peter Waldenberger/Salzburg (Austria)

\section{Participating professional associations and working groups \\ $\nabla$}

AGE, Arbeitsgemeinschaft Gynäkologische Endoskopie der DGGG (Gynecological Endoscopy Working Group of the German Society of Gynecology and Obstetrics)

AGO, Arbeitsgemeinschaft für Gynäkologische Onkologie der DGGG (Gynecological Oncology Working Group of the German Society of Gynecology and Obstetrics)

Berufsverband der Frauenärzte e.V. (Professional Association of Gynecologists)

DeGIR, Deutsche Gesellschaft für Interventionelle Radiologie und minimal-invasive Therapie (German Society for Interventional Radiology and Microinvasive Therapy)

ÖGIR, Österreichische Gesellschaft für Interventionelle Radiologie (Austrian Society for Interventional Radiology)

DRG, Deutsche Röntgengesellschaft (German Society of Radiology)

SSVIR, Swiss Society of Vascular and Interventional Radiology 